\title{
Análise dos fatores de risco relacionados à incidência de infecção do sítio cirúrgico em gastrocirurgias
}

\author{
ANALYSIS OF RISK FACTORS RELATED TO THE INCIDENCE OF SITE INFECTIONS IN GASTROSURGERIES \\ ANÁLISIS DE LOS FACTORES DE RIESGO RELACIONADOS ALA INCIDENCIA DE INFECCIÓN DEL SITIO \\ QUIRÚRGICOEN GASTROCIRUGÍAS
}

\author{
Vanessa de Brito Poveda', Cristina Maria Galvão², Miyeko Hayashida ${ }^{3}$
}

\begin{abstract}
RESUMO
A infecção hospitalar é considerada um problema grave, crescendo tanto em incidência quanto em complexidade, gerando diversos tipos de implicações sociais e econômicas. A presente investigação teve como objetivo identificar a incidência de infecção do sítio cirúrgico (ISC) e os fatores de risco de pacientes submetidos a cirurgias eletivas, na especialidade de Gastroenterologia, realizadas em um hospital público do interior paulista. Os dados foram coletados por meio de estudo retrospectivo de prontuários médicos, no periodo compreendido entre janeiro a dezembro de 1999.

Em 134 casos estudados, detectamos a ocorrência de infecção do sítio cirúrgico em 18 situações $(13,4 \%)$, ocorrendo 9 casos (50\%) do tipo considerado infecção incisional superficial, 8 (44.4\%) infecção incisional profunda e 1 (5.5\%) infecção de órgão/ espaço. Em relação aos fatores de risco presentes nos casos de ISC detectados, os que atingiram porcentagem maior ou igual a 50\% foram: idade acima de 50 anos, presença de neoplasias, dura-ção da cirurgia maior que duas horas e tricotomia inadequada.
\end{abstract}

\section{PALAVRAS-CHAVE}

Infecção hospitalar. Fatores de risco. Cirurgia.

\author{
SUMMARY \\ Nosocomial infection is \\ regarded as a serious problem \\ whose incidence and \\ complexity has been \\ increasing, thus generating \\ various types of social and \\ economic implications. This \\ investigation aimed at \\ assessing the incidence of \\ surgical site infection and risk \\ factors in patients submitted to \\ elective surgeries in the \\ specialty of gastroenterology \\ performed in a public hospital \\ in the interior of São Paulo \\ State. Data were collected by \\ means of a retrospective study \\ of medical records from \\ January to December 1999. Of \\ the 134 cases under study, the \\ occurrence of surgery site \\ infection was detected in 18 \\ situations (13.4\%). Nine cases \\ (50\%) were considered to be \\ superficial incisional \\ infections, 8 (44.4\%) were \\ deep incisional infections and \\ $1(5.5 \%)$ was an organ/space \\ infection. With respect to the \\ risk factors, the ones with \\ percentage higher or equal to \\ $50 \%$ were: age above 50 \\ years, the presence of \\ neoplasia, surgeries that last \\ more than two hours and \\ inadequate trichotomy.
}

\section{KEYWORDS}

Cross infection. Risk factors. Surgery.

\begin{abstract}
RESUMEN
La infección hospitalaria es considerada un problema grave, creciendo tanto en incidencia como en complejidad, generando diversos tipos de implicaciones sociales y económicas. $\mathrm{La}$ presente investigación tuvo como objetivo identificar la incidencia de infección del sitio quirúrgico en pacientes sometidos a cirugias electivas, en la especialidad de Gastroenterología, realizadas en un hospital público del interior del estado de São Paulo. Los datos fueron recoleccionados por medio de un estudio retrospectivo de los archivos medicos, en el periodo entre enero y diciembre de 1999. En 134 casos estudiados, detecta-mos la ocurrencia de infección del sitio quirúrgico en 18 situaciones (13.4\%), ocurriendo 9 casos (50\%) del tipo considerado infección incisional superficial, 8 (44.4\%) infección incisional profunda y 1 (5.5\%) infección de órganolespacio. Con relación a los factores de riesgo presentes en los casos de ISC detectados, los que alcan-zaron percentaje mayor or igual a 50\% fueron: edad acima de 50 años, presencia de neoplasias, duración de la cirugía mayor que dos horas y tricotomía inadecuada.
\end{abstract}

PALABRAS-CLAVE

Infección hospitalaria. Factores de riesgo.Cirugía.
1 Enfermeira. Aluna do Programa de PósGraduação do Departamento de Enfermagem Geral e Especializada da EERP-USP; bolsista do Programa Especial de Treinamento da

Secretaria de Educação Superior (1999-2001)

2 Professora Doutora do Departamento de Enfermagem Geral e Especializada da EERP-USP crisgal@eerp.usp.br

3 Enfermeira. Doutora em Enfermagem Fundamental. Especialista em Laboratório da EERP-USP 
Vanessa de Brito Poveda Cristina Maria Galvão Miyeko Hayashida

\section{INTRODUÇÃO}

O Ministério da Saúde, em consonância com outros órgãos oficiais internacionais, conceitua infecção hospitalar como "aquela adquirida após a admissão do paciente, e que se manifesta durante a internação ou após a alta, quando puder ser relacionada com a internação ou procedimentos hospitalares". ${ }^{(1)}$

Atualmente no Brasil e no mundo a infecção hospitalar (IH) é considerada um problema grave, crescendo tanto em incidência quanto em complexidade, gerando diversos tipos de implicações sociais e econômicas. Em 23 milhões de procedimentos anuais, nos Estados Unidos da

América (EUA), cerca de 920 mil pacientes acabam por desenvolver infecção do sítio cirúrgico (ISC) o que economicamente significa cerca de 10 dias a mais de hospitalização, o que somaria aproximadamente US\$7.500 de custo extra. ${ }^{(2-3)}$

O Centers for Disease Control and Prevention (CDC) dos EUA recomenda que devemos utilizar o termo infecção do sítio cirúrgico em substituição ao infecção da ferida cirúrgica, visto que "nem toda infecção relacionada a manipulação cirúrgica ocorre na ferida propriamente dita, mas também em órgãos ou espaços abordados durante a operação", e pode desenvolver-se até 30 dias após a realização do procedimento cirúrgico e até um ano após, em caso de implante de prótese ou a retirada da mesma. ${ }^{(4)}$

Sabe-se hoje que a ISC é a segunda em termos de incidência, sendo superada apenas pela infecção do trato urinário A ISC pode ser dividida em infecção incisional superficial quando acomete apenas pele ou tecido celular subcutâneo do local da incisão; infecção incisional profunda ao envolver estruturas profundas da parede, a fáscia e a camada muscular; infecção do órgão/espaço, que "envolve qualquer parte da anatomia aberta ou manipulada durante o procedimento cirúrgico com exceção da incisão de parede". ${ }^{(3)}$

Diversos fatores têm sido relacionados à incidência de ISC, como aqueles referentes ao microrganismo, tais como o tamanho do inóculo, reconhece que quanto maior o inóculo maior a chance de ocorrer infecção; fatores relacionados ao paciente como a idade, doenças pré-existentes (diabetes mellitus, obesidade), período longo de hospitalização pré-operatória, desnutrição e fatores relacionados ao procedimento cirúrgico como por exemplo a tricotomia, a presença de drenos e a técnica cirúrgica. ${ }^{(3)}$

A Associação Paulista de Estudos e Controle de Infecção Hospitalar, fundamentada no projeto SENIC (Study on the Efficacy of Nosocomial Infection Control), desenvolvido pelo CDC, enfatiza que o estudo dos aspectos relacionados à incidência, morbiletalidade e custos da ISC, poderiam auxiliar na prevenção em até $20 \%$ através da vigilância epidemiológica associada a implementação de programas de controle. ${ }^{(4)}$

Frente ao exposto, entendemos ser de extrema relevância que os profissionais de saúde compreendam os fatores que influenciam a incidência de ISC para implementarem ações que possibilitem minimizar os riscos de infecções, contribuindo para a melhoria da qualidade da assistência prestada ao paciente cirúrgico e aos profissionais do controle de IH(vinculados a Comissão de Controle de $\mathrm{IH}$ ) o acesso a estudos que ofereçam subsídios que possibilitem reflexões sobre a atuação destes frente as medidas de prevenção e controle de IH.Assim, o presente estudo tem como objetivo identificar a incidência de ISC e os fatores de risco de pacientes submetidos a cirurgias eletivas de gastrectomia, colecistectomia e esofagectomia, na especialidade de Gastroenterologia, de um hospital público do interior paulista.

\section{PROCEDIMENTO METODOLÓGICO}

Após aprovação pelo órgão de documentação médica do hospital investigado, obtivemos uma listagem de todas as cirurgias realizadas durante o ano de 1999, na especialidade de Gastroenterologia, estes dados iniciais foram refinados até chegarmos aos pacientes submetidos a cirurgias eletivas de gastrectomia, colecistectomia e esofagectomia. Os dados foram coletados por meio de levantamento e estudo retrospectivo dos prontuários médicos, no período compreendido entre janeiro a dezembro do ano de 1999 , o que totalizou 134 sujeitos.

Para a coleta dos dados utilizamos um instrumento (ANEXO 1), no qual foi realizada a validação aparente e de conteúdo por três 
especialistas (dois docentes de universidade pública e um profissional de saúde atuante na área de infecção hospitalar), em seguida realizou-se um teste piloto com 16 prontuários médicos para a adequação do instrumento frente ao objetivo proposto. A coleta de dados foi realizada durante o transcorrer de 2000 .

Os autores da presente investigação, tendo como eixo norteador a definição de ISC, buscaram nos prontuários médicos as informações relacionadas ao procedimento cirúrgico até 30 dias após a sua realização.Constatamos que a detecção dos casos de infecções ocorreu durante o período de internação ou no retorno do paciente no ambulatório(previsto de sete a dez dias após o procedimento cirúrgico.No hospital investigado não é realizado o acompanhamento do paciente após a alta hospitalar.Assim, entendemos que os dados disponíveis acarretaram limitações no presente estudo com possibilidade de subnotificação de casos de infecção do sítio cirúrgico.

Ressaltamos que na fase de coleta de dados enfrentamos problemas relativos às informações contidas nos prontuários médicos, como a falta ou escassez de dados. Assim, no instrumento utilizado existem informações que não foram possíveis de serem coletadas acarretando limitações para a análise realizada no presente estudo.

Os resultados foram analisados de forma descritiva e matemático-estatística, utilizando-se freqüência absoluta e porcentagem.

\section{RESULTADOS E DISCUSSÃO}

\section{Fatores relacionados ao paciente}

Na Tabela 1, observamos que a amostra é composta por 79 mulheres (59\%) e 55 homens (41\%). A faixa etária de 51 a 60 anos é a que mais se destaca $(21,6 \%)$ seguida pela de 41 a 50 anos $(19,4 \%)$. Assim, percebemos a predominância de adultos na amostra estudada, embora atualmente a idade não deva ser considerada como fator de risco isolado e sim dentro de todo o contexto como um fator de risco moderado. ${ }^{(3)}$

A ocupação que mais se destaca é aquela ligada a serviços domésticos (42\%); aqui vale lembrar que a amostra é predominantemente feminina. Em seguida, encontramos os aposentados, inativos ou desempregados (16\%) o que também reforça os dados da Tabela 1 , já que $32,8 \%$ dos pacientes cirúrgicos têm mais de 60 anos.

O diagnóstico médico principal mais freqüente foi o de colecistite crônica calculosa, que ocorreu em 71 dos casos; isto corresponde a $52,9 \%$ da amostra em estudo, em segundo lugar as neoplasias, considerando as gástricas, pâncreas e vesícula, com 36 casos $(26,8 \%)$. Em um estudo sobre pacientes portadores de neoplasias, os autores não conseguiram obter dados estatisticamente relevantes indicando a quimioterapia e a radioterapia como fator de risco para o surgimento de ISC, demostraram, porém, que o risco para estes indivíduos está mais ligado a fatores individuais, como a obesidade por exemplo. ${ }^{(5)}$

Tabela 1- Distribuição dos pacientes submetidos à cirurgia na especialidade de gastroenterologia de um hospital público, segundo as variáveis idade e sexo. Ribeirão Preto, 1999

\begin{tabular}{|c|c|c|c|c|}
\hline Sexo & \multirow[t]{2}{*}{ Masculino } & \multirow[t]{2}{*}{ Feminino } & \multicolumn{2}{|c|}{ Total } \\
\hline Idade (anos) & & & $\mathbf{N}$ & $\%$ \\
\hline $0|--| 10$ & 1 & 3 & 4 & 3,0 \\
\hline $11|-| 20$ & 1 & 0 & 1 & 0,8 \\
\hline $21|--| 30$ & 3 & 5 & 8 & 6,0 \\
\hline $31|-| 40$ & 6 & 16 & 22 & 16,4 \\
\hline $41|--| 50$ & 14 & 12 & 26 & 19,4 \\
\hline $51|-| 60$ & 12 & 17 & 29 & 21,6 \\
\hline $61|-|$\begin{tabular}{l|l} 
&
\end{tabular} & 10 & 12 & 22 & 16,4 \\
\hline $71|--| 80$ & 6 & 11 & 17 & 12,7 \\
\hline $81 \mid--$ & 2 & 3 & 5 & 3,7 \\
\hline Total & 55 & 79 & 134 & 100,0 \\
\hline
\end{tabular}


Vanessa de Brito Poveda Cristina Maria Galvão Miyeko Hayashida
Em relação a presença de outras doenças crônicas concomitantes 68 pacientes não apresentaram nenhum tipo de doença $(50,7 \%)$. A doença crônica mais freqüente foi a hipertensão arterial sistêmica em 27 pacientes $(25,1 \%)$, seguida pelo diabetes mellitus com 11 pacientes $(8,2 \%)$, a combinação destas duas patologias ocorreu em 11 casos, representando 8,2\%. Estudiosos demonstraram que a manutenção dos níveis de glicose sanguínea menor que 200 $\mathrm{mg} / \mathrm{dl}$ no pós-operatório imediato reduz a incidência de ISC, uma vez que a função fagocitária das células brancas fica prejudicada em presença de elevados níveis de glicose sangüínea, ou seja, a capacidade de defesa nestes indivíduos está prejudicada expondo-os a uma maior freqüência de infecções agudas ${ }^{(6)}$.

Detectamos que a maioria dos sujeitos não era etilista $(69,4 \%)$, e um número elevado de tabagistas (33,6\%). Em relação aos etilistas atuais, a maioria refere a ingestão de álcool entre 10 e 20 anos, combinações de bebidas fermentadas e destiladas; os tabagistas fumam de 14 a 20 anos, cerca de 20 cigarros por dia.

O álcool é um importante fator de risco para a ISC e deve ser levado em consideração ao se determinar a susceptibilidade individual do paciente, ${ }^{(7)} \mathrm{o}$ tabagismo é realmente um fator de risco para ISC em cirurgias que envolvam o esterno e o mediastino, embora estudos existentes a este respeito utilizem como unidade de medida as categorias de "fumantes ativos" ou "correntes", não obtendo desta forma a determinação da quantidade em números absolutos do tabaco como risco para $\mathrm{ISC}^{(8)}$. Estudiosos observaram a maior incidência de infecção nos casos de fumantes submetidos a cirurgias bucomaxilares ${ }^{(9)}$; não encontramos estudos decisivos sobre a contribuição do tabaco em cirurgias de outras especialidades.

Tabela 3- Distribuição dos pacientes submetidos à cirurgia na especialidade de gastroenterologia de um hospital público, segundo o tipo de cirurgia realizada. Ribeirão Preto, 1999.
O tempo de hospitalização pré-operatória variou entre 1 e 33 dias, sendo que 92 pacientes $(68,6 \%)$ aguardaram até 5 dias hospitalizados a cirurgia proposta (Tabela 2). De acordo com a literatura, o período de hospita-lização pré-operatória é freqüentemente associado ao aumento do risco do paciente desenvolver ISC, devido a colonização da pele pela característica microbiota hospitalar. Em pacientes com 7 dias de hospitalização prévia, os índices de ISC se tornam até duas vezes superiores aos com apenas 1 dia de internação. ${ }^{(4 ; 8)}$

Tabela 2 - Distribuição dos pacientes submetidos à cirurgia na especialidade de gastroenterologia de um hospital público, segundo os dias de hospitalização préoperatória. Ribeirão Preto, 1999

\begin{tabular}{|c|c|c|}
\hline $\begin{array}{c}\text { Dias de } \\
\text { hospitalização }\end{array}$ & $\mathbf{N}$ & $\%$ \\
\hline $0|--| 5$ & 92 & 68,6 \\
\hline $6|--| 10$ & 25 & 18,6 \\
\hline $11|--| 15$ & 9 & 6,7 \\
\hline $16 \mid--20$ & 6 & 4,5 \\
\hline $21|-| 25$ & 1 & 0,8 \\
\hline $26|--| 33$ & 1 & 0,8 \\
\hline Total & 134 & 100,0 \\
\hline
\end{tabular}

\section{Fatores relacionados ao procedimento cirúrgico}

A Tabela 3 mostra que 91 pacientes foram submetidos a colecistectomia $(67,9 \%)$, a gastrectomia foi a segunda cirurgia mais freqüente com 20 casos (15\%).

\begin{tabular}{lcc}
\hline \multicolumn{1}{c}{ Tipo de cirurgia } & N & \% \\
\hline Colecistectomia & 91 & 67,9 \\
Esofagectomia & 10 & 7,5 \\
Gastrectomia & 20 & 15,0 \\
Gastrectomia + Colecistectomia & 9 & 6,7 \\
Gastrectomia + Esofagectomia & 3 & 2,2 \\
Gastrectomia + Esofagectomia + Colecistectomia & 1 & 0,7 \\
\hline \multicolumn{1}{c}{ Total } & 134 & 100,0 \\
\hline
\end{tabular}


Em relação à duração do procedimento cirúrgico, observamos que 49 pacientes (36,6\%), tiveram tempo cirúrgico entre 15 e 120 minutos; 47 pacientes $(35,1 \%)$, com 121 a 240 minutos; seguido por 31 casos $(23,1 \%)$, com duração de 241 a 360 minutos (Tabela 4). Existe acréscimo no risco de ISC em cada hora a mais de cirurgia, embora este mesmo risco não possa ser muito bem esclarecido por facilmente ser confundido ou estar acompanhado por outros. ${ }^{(10)}$

Em 36 situações (26,9\%) drenos foram utilizados e independente do tipo representam fator de risco, predispondo o paciente à infecção; sendo que os indivíduos portadores apresentam risco de desenvolver infecção por 15 dias, enquanto na ausência destes o risco é de 9 dias; se o sistema de drenagem for aberto os índices de ISC podem chegar até a $15,7 \%$, comparados a $10,1 \%$ entre aqueles que possuem sistema de drenagem fechado. Cirurgiões mesmo cientes do perigo, partilham de uma opinião prevalente que a coleção de fluidos no local da incisão predispõe os pacientes a infecções maiores e mais extensas do que a que poderia causar o dreno, vale ressaltar também a combinação dos fatores de risco, pois drenos são freqüentemente mais utilizados em cirurgias longas e indivíduos submetidos a estas, possuem doenças de base mais avançadas. ${ }^{(11)}$

O dreno representa uma porta de entrada para microrganismos e a sua colocação deve ser realizada após uma avaliação criteriosa, considerando a relação risco x benefício. Recomenda-se o uso deste com sistema fechado de drenagem, cuidados rigorosos na sua manipulação e a permanência a mais curta possível ${ }^{(3-4)}$.

A tricotomia foi realizada em 51 situações, sendo predominantemente abdominal (43 casos), em 10 casos foi realizada até 12 horas antes da cirurgia, em 30 casos de 12 a 24 horas antes do procedimento, com mais de 24 horas, foram 11 casos. A tricotomia vem sendo há muitos anos discutida e é sabido por meio de estudos, que a remoção do pêlo com lâmina da região a ser operada, na noite anterior à cirurgia é associada com um significativo aumento do risco de ISC, do que o uso de agentes depilatórios ou a não remoção do pêlo; quanto menor o tempo entre a tricotomia com lâmina e a cirurgia decresce a taxa de $\operatorname{ISC}^{(8)}$. Atualmente, recomenda-se a realização de tricotomia imediatamente antes da cirurgia (prazo máximo de duas horas) e se estritamente necessária, com a utilização preferencialmente de tricotomizador elétrico. No presente estudo, o procedimento em questão foi realizado com lâmina e na maioria dos casos (40) dentro das 24 horas que antecederam a cirurgia, ou seja, de forma inadequada do preconizado pela literatura.

A anti-sepsia foi realizada com polivinilpirrolidona iodo alcoólica (PVPI) em 116 situações $(86,6 \%)$, com tintura de merthiolate em 15 situações (11,1\%), e 3 com PVPI aquoso $(2,2 \%)$. A preparação da pele com anti-sépticos visa minimizar que microrganismos existentes na microbiota da pele sejam levados para dentro da ferida operatória durante a incisão e manipulação dos tecidos. Embora o uso de clorexidina a 4\% elimine mais bactérias gram negativas do que outros produtos e tenha uma maior ação residu$\mathrm{al}^{(8,12)}$, neste estudo o uso foi predominantemente do PVPI alcoólico, apesar de existirem estudos que demonstram a resistência de microrganismos, como o Stafilococcus aureus meticilino resistente, ${ }^{(13)}$ e que este pode ser inativado pelo sangue ou proteínas séricas, mas exerce um efeito bacteriostático tão longo quanto esteja presente na pele. ${ }^{(8)}$

No presente estudo, verificamos que em 60 situações $(44,7 \%)$, os pacientes foram submetidos a sondagem vesical de demora e nasogástrica, 27 situações $(20,1 \%)$ submetidos a sondagem vesical de demora, 11 situações $(8,2 \%)$ submetidos a sondagem nasogástrica e 10 situações $(7,4 \%)$ submetidos a sondagem vesical e nasoenteral. Ressaltamos que a pele e mucosas são barreiras naturais de defesa do organismo humano,

Tabela 4- Distribuição dos pacien-tes submetidos à cirurgia na especialidade de gastroenterologia de um hospital público, segundo a duração do procedimento cirúrgico. Ribeirão Preto, 1999.

\begin{tabular}{|c|c|c|}
\hline $\begin{array}{c}\text { Duração da cirurgia } \\
\text { (minutos) }\end{array}$ & $\mathbf{N}$ & $\%$ \\
\hline $15|--| 120$ & 49 & 36,6 \\
\hline $121|-| 240$ & 47 & 35,1 \\
\hline $241|--| 360$ & 31 & 23,1 \\
\hline $361|-| 480$. & 4 & 3,0 \\
\hline 481 |- & 3 & 2,2 \\
\hline Total & 134 & 100,0 \\
\hline
\end{tabular}

Análise dos fatores de risco relacionados à incidência de infecção do sítio cirúrgico em gastrocirurgias 
Vanessa de Brito Poveda Cristina Maria Galvão Miyeko Hayashida juntamente com os processos de remoção mecânica de microrganismos, tais como a sudorese, peristaltismo, salivação, dentre outros; a introdução de cateteres acarreta quebra destas barreiras propiciando o desenvolvimento de infecção ${ }^{(14)}$.

A antibioticoprofilaxia (ATBP) foi realizada em todos os casos, na indução anestésica. Estudiosos ressaltam que existem 4 princípios básicos para maximizar os benefícios desta conduta:

- usar ATBP quando comprovadamente mostre redução dos índices de ISC;

- usar um antibiótico que seja seguro, barato e com espectro bactericida in vitro que cubra os mais prováveis contaminantes intraoperatórios;

- tempo de infusão da dose inicial do antibiótico de maneira que a concentração bactericida da droga seja estabilizada no sangue e nos tecidos ao mesmo tempo em que a pele é incisionada;

- manutenção dos níveis terapêuticos de agentes antimicrobianos no sangue e tecidos durante a cirurgia e até poucas horas após a incisão ser fechada na sala operatória. ${ }^{(8)}$

\section{Fatores relacionados à incisão cirúrgica}

Em 134 casos estudados, detectamos a ocorrência de infecção do sítio cirúrgico em 18 situações $(13,4 \%)$; esta porcentagem está próxima da faixa aceitável para cirurgias potencialmente contaminadas que é de 3 a 11\%; a partir desta taxa as contaminações exógenas são o principal fator para o desenvolvimento das infecções cirúrgicas. ${ }^{(3)}$
A distribuição dos casos de infecção foi classificada, segundo o tipo, de acordo com os critérios definidos pelo National Nosocomial Infections Surveillance (NNIS) do $\mathrm{CDC}^{\left({ }^{(8)}\right.}$. Considerando-se o total de casos com presença de ISC $(n=18)$ observamos que 9 casos $(50 \%)$ correspondem a infecção considerada incisional superficial, seguida pela infecção incisional profunda com 8 situações $(44,4 \%)$ e apenas $1(5,5 \%)$ considerada de órgão e/ou espaço.

Em um estudo detectou-se também maior incidência de infecção considerada incisional superficial, os autores da investigação ressaltaram a importância da vigilância pós-alta, pois $32,5 \%$ dos casos de infecção detectados foram identificados no ambulatório durante o retorno do paciente; entretanto, não constava no artigo qual o período de acompanhamento do paciente após a realização do procedimento cirúrgico. ${ }^{(15)}$

$\mathrm{Na}$ Tabela 5 demonstramos os fatores de risco de infecção do sítio cirúrgico encontrados nos 18 casos de infecção detectados neste estudo.

A Tabela 5 aponta que para a maioria dos pacientes que manifestaram ISC, houve a presença de fatores de risco como: a idade acima de 50 anos $(66,7 \%)$, neoplasias $(55,6 \%)$, tricotomia inadequada $(55,6 \%)$ e a duração da cirurgia por mais de 120 minutos $(83,3 \%)$.

Na presente investigação, do total de casos que apresentaram infecção, $10(55,6 \%)$ são do sexo masculino e $8(44,4 \%)$ do feminino. Entretanto, não foi possível estabelecer uma ligação entre sexo e a maior probabilida-

Tabela 5- Distribuição dos pacientes que apresentaram infecção do sítio cirúrgico na especialidade de gastroenterologia de um hospital público, segundo a duração do procedimento cirúrgico. Ribeirão Preto, 1999.

\begin{tabular}{lcccccc}
\hline \multirow{2}{*}{ Fatores de risco } & \multicolumn{2}{c}{ Sim } & \multicolumn{2}{c}{ Não } & \multicolumn{2}{c}{ TOTAL } \\
\cline { 2 - 7 } & $\mathbf{N}$ & \% & N & \% & N & $\%$ \\
\hline Idade > 50 anos & 12 & 66,7 & 6 & 33,3 & 18 & 100,0 \\
Tabagismo & 6 & 33,3 & 12 & 66,7 & 18 & 100,0 \\
Etilismo & 8 & 44,4 & 10 & 55,6 & 18 & 100,0 \\
Neoplasia & 10 & 55,6 & 8 & 44,4 & 18 & 100,0 \\
Diabetes Mellitus & 4 & 22,2 & 14 & 77,8 & 18 & 100,0 \\
Pré-hosp. > 5 dias & 8 & 44,4 & 10 & 55,6 & 18 & 100,0 \\
Duração cir. > 120' & 15 & 83,3 & 3 & 16,7 & 18 & 100,0 \\
Drenos & 8 & 44,4 & 10 & 55,6 & 18 & 100,0 \\
Tricotomia inadequada & 10 & 55,6 & 8 & 44,4 & 18 & 100,0 \\
Anti-sepsia merthiolate & 2 & 11,1 & 16 & 88,9 & 18 & 100,0 \\
\hline
\end{tabular}


de de desenvolver ISC, como em um estudo, no qual houve moderada prevalência de ISC em mulheres submetidas a cirurgias cardíacas. ${ }^{(16)}$

Sabemos, de acordo com o exposto anteriormente, que a realização de tricotomia inadequada é um fator que aumenta o risco de ISC, assim como o uso indiscriminado de drenos. Já em relação as neoplasias, a literatura é controversa e não elucidativa, sugerindo a necessidade de mais estudos a este respeito.

Ressaltamos que o uso de merthiolate para anti-sepsia da pele do paciente na instituição investigada consiste em prática da equipe médica; entretanto esta solução não é recomendada pela literatura ${ }^{(4)}$. A partir desta análise, entendemos que houve quebra da assepsia cirúrgica e consideramos como fator que poderia relacionar-se com a ISC detectada.

\section{CONCLUSÕES}

O presente estudo possibilitou as seguintes conclusões:

- a taxa de incidência de ISC encontrada no estudo foi de $13,4 \%$ (18/134), taxa próxima da aceitável para cirurgias potencialmente contaminadas;

- em relação ao tipo de ISC, detectou-se 9 casos $(50 \%)$ categorizados como infecção considerada incisional superficial, 8 casos
(44,4\%) considerados infecção incisional profunda e apenas $1(5,5 \%)$ considerado infecção de órgão e/ou espaço;

- dentre os fatores de risco presentes nos casos de ISC detectados, os que atingiram porcentagem ${ }^{3} 50 \%$ foram: idade acima de 50 anos, presença de neoplasias, duração da cirurgia maior que 2 horas e tricotomia inadequada.

Os gastos da previdência social com o paciente que desenvolve ISC e que este se hospitaliza novamente para tratamento das seqüelas é acentuado, gerando custos elevados para a instituição hospitalar. ${ }^{(17)}$

Os profissionais de saúde devem ter em mente a magnitude das implicações sociais, econômicas e pessoais devido a IH, implicações que atingem tanto o paciente, afastado de sua família e trabalho produtivo por mais tempo, como para a instituição hospitalar e para o país.

Ao nosso ver, fica claro a importância de medidas para a prevenção e controle da ISC e salientamos aqui o papel fundamental da enfermagem no período perioperatório. Assim, acreditamos que este estudo oferece subsídios para novas pesquisas nacionais que elucidem melhor a contribuição dos fatores predisponentes para ISC, pois compete ao enfermeiro perioperatório promover uma assistência de enfermagem direcionada ao atendimento das reais necessidades do paciente, minimizando os riscos e complicações inerentes ao procedimento anestésico cirúrgico.

\section{REFERÊNCIAS}

(1) Brasil. Ministério da Saúde. Portaria no 2616, de 12 de maio de 1998. Aprova programa de controle de infecção hospitalar e dá outras providências. Diário Oficial da República Federativa do Brasil, Brasília, 13 maio 1998. Seção 1, p.133-5.

(2) Wenzel RP. Preoperative antibiotic prophylaxis. N Engl J Méd 1992; 5: 337-9.

(3) Rabhae GN, Ribeiro-Filho N, Fernandes AT. Infecção do sítio cirúrgico. In: Fernandes AT, Fernandes MOV, Ribeiro-Filho, N. Infecção hospitalar e suas interfaces na área da saúde. São Paulo: Atheneu; 2000, p. 479-505.

(4) Associação Paulista de Estudos e Controle de Infecção Hospitalar. Prevenção da infecção de sítio cirúrgico. São Paulo (SP); 2001.
(5) Barber GR, Miransky J, Brown AE, Coit DG, Lewis FM, Thaler HT et al. Direct observations of surgical wound infections at a comprehensive cancer center. Arch Surg 1995; 130:1042-7.

(6) Zerr KJ, Furnary AP, Grunkemeier GL, Bookin S, Kanhere V, Starr A. Glucose control lowers the risk of wound infection in diabetics after open heart operations. Ann Thorac Surg 1997; 63:356-61.

(7) Rantala A, Lehtonen OP, Niinikoski J. Alcohol abuse: a risk factor for surgical wound infections? Am J Infect Control 1997; 25:381-6.

(8) Mangram AJ, Horan TC, Pearson ML, Silver LC, Jarvis WR. Guideline for prevention of surgical site infection, 1999. Am J Infect Control 1999; 27:97-132.
Análise dos fatores de risco relacionados à incidência de infecção do sítio cirúrgico em gastrocirurgias 
Vanessa de Brito Poveda Cristina Maria Galvão Miyeko Hayashida
(9) Jones JK, TriplettRG. The relationship of a cigarette smoking to impaired intraoral wound healing: a review of evidence and implications for patient care. J Oral Maxillofac Surg 1992; 50: 237-9.

(10) Vilar-Compte D, Mohar A, Sandoval S, Rosa M, Gordillo P, Volkon P. Surgical site infections at the National Cancer Institute in Mexico: a case control study. Am J Infect Control 2000; 28:14-20.

(11) Simchen E, Rozin R, Wax Y. The Israeli study of surgical infection of drains and the risk of wound infection in operations for hernia. Surg Gynecol Obstet 1990; 170:331-7.

(12) Faoagali JL, George N, Fong J, Davy J, Dowser M. Comparison of the antibacterial efficacy of $4 \%$ clhorhexidine gluconate and $1 \%$ triclosan handwash products in a acute clinical ward. Am J Infect Control 1999; 27:320-6.

(13) Jones RD. Bacterial resistance and topical antimicrobial wash products. Am J Infect Control 1999; 27:351-63.
(14) Lacerda RA, editor. Buscando compreender a infecção hospitalar no paciente cirúrgico. São Paulo: Atheneu; 1992.

(15) Lemos FN, Oliveira AV, Sousa MG. Infecção de sítio cirúrgico: estudo prosprectivo de 2149 pacientes operados. Rev Col Bras Cirurgiões 1999; 26:109-13.

(16) Lilienfield DE, Vlahov D, Tenney, JH, McLaughlin JS. Obesity and diabetes as risk factors for postoperative wound infections after cardiac surgery. Am J Infect Control 1998; 16:3-6.

(17) Poulsen KB, Bremmelgaard A, Sorensen AI, Raahave D, Petersem IV. Estimated costs of postoperative wound infections: a casecontrol study of marginal hospital and social security costs. Epidemiol Infect 1994; 113: 283-295. 


\section{FATORES RELACIONADOS AO PACIENTE}

$\mathrm{N}^{\circ}$ do prontuário

Idade

Sexo ( )M ( )F

Profissão..

Diagnóstico médico

Data da internação

Data da cirurgia

Data da alta

Presença de doenças crônicas

Desnutrição ( ) Peso:

Altura:

$\mathrm{IMC}$ :

Etilismo ( ) Freqüência:

Tabagismo ( ) Freqüência:

Uso de esteróides ( ) Especificar:

Infecção preexistente ( ) Especificar:

\section{FATORES RELACIONADOS AO PROCEDIMENTO CIRÚRGICO}

Antibioticoprofilaxia ( )

Data de início: .........../.... Data de término: ................

Especificar (medicamento/dosagem):

Durante o procedimento cirúrgico ( )

Indução anestésica ( )

Outro( ) Especificar:

Antibioticoterapia ( )

Data de início: .................

Especificar (medicamento/dosagem):

Cirurgia proposta:

Cirurgia realizada:

Início: Término:

Anestesia:

Início: Término:

Tricotomia ( ) Data: ................. Horário:

Local: ......

Anti-sepsia da pele ( ) PVPI ( ) alcoólico ( ) aquoso

( ) clorohexidina

( ) outro Especificar:

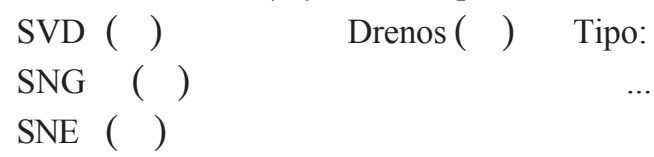

\section{FATORES RELACIONADOS A INCISÃO CIRÚRGICA}

Infecção do sítio cirúrgico

Tipo superficial profunda órgão/espaço (cavidade)

Caracterização descrita no prontuário médico:

Tipo de microrganismo isolado (em caso de cultura): 